\title{
Spatial and electronic correlations in the PE545
}

\section{light-harvesting complex}

\author{
Lucas Viani ${ }^{\dagger *}$, Carles Curutchet ${ }^{t^{*}}$, and Benedetta Mennucci ${ }^{\dagger^{* *}}$
}

$\dagger$ Dipartimento di Chimica e Chimica Industriale, Universitá di Pisa, via Risorgimento 35, 56126 Pisa, Italy.

†Departament de Fisicoquímica, Facultat de Farmàcia, Universitat de Barcelona, Av. Joan XXIII s/n, 08028 Barcelona, Spain.

KEYWORDS : light harvesting, pigment-protein complexes, quantum coherence, electronic energy transfer.

ABSTRACT: Recent discovery of long-lasting quantum coherence effects in photosynthetic pigment-protein complexes have challenged our view of the role that protein motions play in light harvesting processes. Several groups have suggested that correlated fluctuations involving the pigments site energies and couplings could be at the origin of such unexpected behavior. Here we combine molecular dynamics simulations with quantum mechanics/molecular mechanics calculations to analyze the degree of correlated fluctuations in the PE545 complex of Rhodomonas sp. strain CS24. We find that correlations between the motions of the chromophores, which are significantly assisted by the water solvent, do not translate into appreciable site energy correlations, but do lead to significant cross-correlations of energies and couplings. Such behavior, not observed in a recent study on the Fenna-Mathews-Olson complex, seems to provide phycobiliproteins with an additional fundamental mechanism to control quantum coherence and light harvesting efficiency compared to chlorophyll-containing complexes. 
In the initial steps of photosynthesis, special antenna proteins composed of a large numbers of light-absorbing molecules (pigments) are used to harvest photons and transfer their energy to reaction centers, where it is used to drive charge separation events. ${ }^{1,2}$ There is a rich variety of pigment-protein antenna complexes adapted to the light quality of their habitats, ${ }^{3}$ in which absorbed photons are funneled to reaction centers with very high quantum efficiencies. It is highly desirable to achieve similar performances in artificial photosynthetic devices inspired on this same principle. ${ }^{4,5}$ In this quest for efficient artificial light-harvesting devices, a comprehensive understanding of light-harvesting dynamics and their relation to molecular structure is a key aspect. ${ }^{5}$ Although much of this understanding has already been achieved over the last decades, ${ }^{1,2}$ recent discoveries of unexpected long-lasting quantum coherence effects in several photosynthetic antenna complexes have challenged our view of the role that protein structure and motions play in the process. ${ }^{6-11}$ In particular, these experiments have suggested that the protein environment protects quantum coherence by providing correlated fluctuations on the pigment's uncoupled transition energies, the site energies. This hypothesis has led to a remarkable research effort aimed at assessing the consequences of correlated fluctuations on quantum coherence and exciton dynamics. ${ }^{12-19}$ Even in the weak coupling regime characterized by Förster theory, bath modes shared between different chromophores can affect electronic energy transfer (EET) dynamics by changing the spectral overlap factor. ${ }^{20}$ More recently, some authors have also addressed the impact of correlations between the fluctuations of site energies and interpigment couplings, or among couplings, suggesting that such additional correlations also modulate quantum coherence and energy transfer dynamics. ${ }^{21,22}$ It is thus of high interest to estimate whether or not energy and coupling fluctuations in photosynthetic antennae are actually correlated and the molecular mechanisms supporting this correlation. 
Olbrich et al. have examined the degree of correlated fluctuations in the peripheral lightharvesting complex 2 (LH2) and in the Fenna-Matthews-Olson (FMO) complex by combining quantum chemistry with classical molecular dynamics (MD) simulations, suggesting that the degree of correlated fluctuations between site energies is indeed small, although correlations in the electronic couplings were observed. ${ }^{23,24}$ Both FMO and LH2 systems contain chlorophylls, pigments that are not covalently bound to the protein scaffold, and recent studies suggest that site energy tuning in chlorophyll-containing complexes is achieved mainly through specific pigmentprotein interactions. ${ }^{25-27}$ Recently, we have shown that in the phycoerythrin 545 (PE545) complex (see Figure1), belonging to a different family of antenna proteins called phycobiliproteins, site energy tuning is mainly achieved by modulating the pigment conformations inside the complex, rather than through specific pigment-protein interactions. ${ }^{28}$ Phycobiliproteins contain special linear tetrapyrrole pigments called bilins, and the rather flexible structure of such molecules seems to explain the fact that site energy tuning is mainly achieved by controlling the pigment conformation. Moreover, bilin pigments are covalently bound through either one or even two covalent bonds to cysteine residues in the protein. Thus, the protein could assist correlated fluctuations in a qualitatively different manner compared to LH2 and FMO. Indeed, significant quantum coherence effects at room temperature were first observed for the PE545 antenna and a similar biliprotein. ${ }^{8}$

In this study, we combine a polarizable quantum mechanical/molecular mechanical (QM/MM) methodology we recently developed, ${ }^{29}$ which we call MMPol, with classical MD simulations in order to assess the degree of correlated fluctuations in the PE545 principal light-harvesting antenna of the cryptomonad Rhodomonas sp. strain CS24. ${ }^{28,30-34}$ In this approach, we first performed a ground-state classical MD simulation of PE545 in water (see Ref. [28] for details), 
followed by QM/MMPol calculations of the EET parameters (site energies and excitonic couplings) along the classical trajectory, allowing us to obtain a series of values of these parameters necessary to estimate potential correlations due to the structural fluctuations of the system. In particular, we extracted 60000 snapshots every 5 fs corresponding to 300 ps of a MD simulation of PE545 for the subsequent QM/MMPol calculations. In addition, we also performed an analogous simulation of the complex in vacuo in order to assess the impact of the solvent in assisting spatial and electronic correlations. Due to the considerable computational cost involved in the QM/MMPol calculations, we adopted the ZINDO semiempirical method with the Zerner's spectroscopic parameterization ${ }^{35}$ to describe the excited states of the pigments, whereas the protein and solvent environment were described using a polarizable force field we recently developed from DFT calculations (see SI). All the details about the QM/MMPol model are reported in Refs. [28,34].

Before moving to analyze the correlations, in Figure 1 we report the distribution of site energies of the various pigments as obtained from the MD-QM/MMPol calculations.

\section{Fig. 1}

As it can be seen from the plot, the fluctuations induced in the site energies by internal motions, as well as by the protein+solvent motions are similar for all pigments, with only the $\mathrm{DBV}_{\mathrm{A}}$ and $\mathrm{DBV}_{\mathrm{B}}$ pigments showing broader bands. It is here important to note that the ZINDO energetic ordering of the pigments is consistent with the energy transfer scheme we recently derived for PE545 using ab initio methods. ${ }^{28}$ 
Commonly, the correlation between one-dimensional sets of data is described using Pearson coefficients. Its multidimensional formulation (see SI) is commonly used in the identification of collective motions in proteins ${ }^{36,37}$ although part of the spatial correlations are underestimated since it is based on the assumption of collinear motion of atoms, and its dependence on the relative orientation of the spatial fluctuations. ${ }^{38}$ One way to avoid all these flaws is the use of approaches based on the mutual information (MI) of the random variables, which relies on the calculation of their entropies and marginal entropies (see SI). ${ }^{39,40}$ The general MI framework, indicated here as GMI, has been first applied to proteins in Ref.[38] where the dependence on the nonlinear motions was identified as the main reason for the underestimation of the atomic spatial correlations predicted by the Pearson coefficients. The impact of the nonlinear contributions in the GMI coefficients can be quantified by confronting it with the so called linear mutual information (LMI) approach. ${ }^{38}$ In this approximation, only the collinear contributions are captured since a Gaussian probability distribution of the random variables is assumed. This approach is similar to the Pearson method although it is not restricted to the relative orientation of the atomic spatial fluctuations. Both MI approaches provide correlation coefficients varying between zero and infinity, with no obvious intuitive interpretation; therefore MI values were rescaled to the $[0.0,1.0]$ range by applying a nonlinear relation, ${ }^{38,40}$ which may result in relatively larger coefficients, especially for the lower ones ${ }^{24}$ All the correlations were calculated using inhouse software.

Based on the configurations extracted from the MD trajectory, the correlation coefficients of the positional fluctuations for each pair of atoms were computed using all three approaches, Pearson, GMI and LMI (see Fig. 2).

\section{Fig.2}


As shown in Figure 2a, the generalized method predicts a stronger correlation between the atomic positional fluctuations in the system, suggesting that a large fraction of the total correlation corresponds to the nonlinear component, as already reported in Ref.[38]. The difference in the magnitude of the mean correlation coefficients obtained with the GMI $(0.66)$, LMI (0.23), and Pearson (0.04) is shown in Figure $2 \mathrm{~b}$ where the dependence of each correlation with the interatomic distance is reported. In contrast to what is observed in the LH2 and FMO complexes, ${ }^{23,24}$ for the PE545 complex a strong correlation $(\sim 0.5)$ is found for atoms with distances larger than $30 \AA$. It is important to highlight that the majority of these atom pairs are anticorrelated. It is also worth noting the good match between the LMI and Pearson distributions for coefficients larger than 0.4: this match indicates that the rescaling artifact introduced in the MI is not affecting the results for large correlations. The absence of long-range spatial correlations in LH2 or FMO, and the large values observed in the PE545 complex may be explained by the covalent bonds between bilins and the protein scaffold, and the solvent mediated correlation, given that the pigments in PE545 are significantly more exposed to the water than the chlorophylls in FMO or LH2.

To evaluate the impact of the solvent in these spatial correlations we applied the same procedure to the MD trajectory obtained for the system in vacuo and then we subtracted the two corresponding correlation coefficients. This was possible since the morphology of the system in vacuo remained comparable to the solvated one during the MD simulation. The RMSD between the crystal structure and the structures of the PE545 complex sampled along the MD simulations in vacuum and in solution amount to $2.5 \AA$ and $1.85 \AA$, respectively. The change in the spatial correlation upon removal of the solvent versus the interatomic distance is shown in Figure 3 for 
the $\mathrm{DBV}_{\mathrm{A}}, \mathrm{PEB}_{158 \mathrm{D}}, \mathrm{PEB}_{82 \mathrm{C}}$, and $\mathrm{PEB}_{50 / 61 \mathrm{D}}$ pigments. The dataset of the remaining pigments are not displayed in the plot since they are similar and yield the same conclusions.

Fig. 3

Although the mean value of the variations (indicated by the dashed lines) are around 0.1 for all pigments, the long distance correlations show a larger sensitivity to the lack of the solvent, featuring a reduction of up to 0.38 in the total correlation coefficient (see the $\mathrm{DBV}_{\mathrm{A}} / \mathrm{DBV}_{\mathrm{B}}$ pair indicated by the red symbols), while for the short distance correlations the maximum reduction is 0.25. In both cases, it is enough to reduce the correlation by at least one half upon removal of the solvent. Therefore the spatial correlations involving $\mathrm{DBV}_{\mathrm{A}}, \mathrm{DBV}_{\mathrm{B}}, \mathrm{PEB}_{158 \mathrm{C}}$, and $\mathrm{PEB}_{158 \mathrm{D}}$ are considerably assisted by the network of water molecules that solvates the complex. Water fluctuations have also been found to induce significant correlations between site energies in hydrated DNA, enhancing the hole transfer dynamics. ${ }^{42}$

To investigate if these spatial correlations can also induce significant electronic correlations, we computed the Pearson coefficients among the fluctuation of site energies and couplings. Figure 4 shows the results obtained using two different descriptions of the couplings, namely the QM/MMPol (QM) scheme (lower triangle), and the point-dipole (PD) approximation assuming no screening of the environment (upper triangle). The lower left rectangle contains the correlations between site energies computed at QM/MMPol level. The results based on the MI approaches are not shown since they do not add any extra information.

\section{Fig. 4}

Despite the significant spatial correlations found between pigments, fig. 4 shows that the site energy correlations are much weaker. This is not completely unexpected as spatial correlations between the positions of individual atoms do not necessarily lead to appreciable site energy 
correlations; in fact a given site energy depends simultaneously on the positions of all the pigment atoms, and to a smaller extent also on the positions of other surrounding atoms through electrostatic interactions. The largest site energy correlation amounts to 0.04 and is observed for the $\mathrm{DBV}_{\mathrm{A}} / \mathrm{DBV}_{\mathrm{B}}$ pair, which has an average center separation of $45 \AA$. It is probably associated with the large spatial correlation between the two molecules (see Figure 2a), as discussed previously. The cross-correlations (site energies-coupling) feature larger values, especially when both are related to the same pigment. In this specific case, the anticorrelation suggests that an increase/decrease on the site energy of a pigment is accompanied by a decrease/increase of the coupling of this pigment with the others. However, these anticorrelations often have different magnitudes and significant consequences could arise on both the coherences and the transfer rates as shown by Silbey and co-workers. ${ }^{15,21}$ These effects may be particularly important in strongly coupled pairs such as $\mathrm{PEB}_{50 / 61 \mathrm{C}}$ and $\mathrm{PEB}_{50 / 61 \mathrm{D}}$, given that the anticorrelation involving the site energy of $\mathrm{PEB}_{50 / 61 \mathrm{C}}$ is clearly larger than that involving $\mathrm{PEB}_{50 / 61 \mathrm{D}}$.

We also note that the results are very similar regardless the method used to estimate the couplings. Considering that no explicit solvent screening effects were taken into account in the PD calculations, the origin of such correlations has its origin in changes of relative position and orientation between the pigments induced by the solvent and protein environment, rather than to induced effects on the electronic interactions. These results are quite in contrast to those obtained by Olbrich and co-workers on the FMO complex, where significant cross-correlations were obtained using the PD approximation, whereas they almost disappeared upon taking into account the shape of the transition densities through the more accurate TrEsp approach. ${ }^{24}$ As the TrEsp approach should describe coupling fluctuations in a similar way compared to our method, we argue that the significant cross-correlations found in PE545 should be related to the different 
nature of bilin chromophores compared to the chlorophylls in FMO. Another possible explanation for this contrasting behavior, however, could be the neglect of fluctuations in the TrEsp charges used in the FMO study, whereas we calculate the transition densities on each structure of the PE545 complex considered, so they can naturally fluctuate. Returning to the different nature of bilins and chlorophylls, and the question why bilin cross-correlations could be stronger, this is probably a consequence of the more flexible structure of bilins compared to chlorophylls: their site energies are very sensitive to the conformation of the flexible linear tetrapyrrole structure, and any small change in the torsional angles affecting the orientation of the pigment, and therefore their coupling to other molecules, would likely translate into a change in the degree of $\pi$ conjugation between the different rings and consequently in its site energy.

Regarding the more obvious correlations between excitonic couplings, Figure 4 shows that both, correlated and anti-correlated pairs are found. It is important to highlight that effects on the exciton dynamics are generally amplified for correlations with opposite signs..$^{21,22}$ Even though, the impact of coupling-coupling correlations is expected to be less pronounced in the coherence when compared to the cross-correlation one, they could also play a significant role in PE545 light-harvesting dynamics. ${ }^{21}$

Finally, to investigate the effects of the polarization of the environment on the correlations, we have performed the same steps calculating the site energies and couplings in a non-polarizable QM/MM scheme. As expected, the results reveal that the polarization is essential in the calculation of the site energies and couplings, although no effects are observed in the correlations (the results are provided in the SI). Once again, this result can be related to the fact that in the PE545 correlations are mainly induced by fluctuations in the environment structural network and not by changes in the local electric fields. 
In summary, while fluctuations and correlations in site-energies have been often proposed as a possible origin of the observed long-lived coherences, here we have shown that these are almost negligible in the PE545 system. On the contrary, we have found significant correlations between the fluctuations of (i) site energies and couplings, and (ii) couplings sharing a pigment. This important finding is in contrast to recent studies on the FMO complex, where correlations involving energies and couplings were found to be negligible, ${ }^{24}$ and appears to be linked to the more flexible nature of bilin pigments compared to chlorophylls. This is coherent with a recent theoretical analysis showing that in PE545, quantized intramolecular vibrations can compete with slow modes arising from pigment-protein interactions ${ }^{43}$ in order to have an efficient energy transport. Moreover, two other recent studies $^{21,22}$ have shown that both energy-coupling and coupling-coupling correlations can lead to important changes in the interpigment transfer rates and coherence, although the effect of cross-correlations should be more pronounced. The increased ability of phycobiliproteins to induce cross-correlations compared to chlorophyllcontaining complexes thus seems to provide the former with an additional mechanism to control quantum coherence and light harvesting efficiency. If the findings of our simulation of the PE545 system and these new findings are really consistent and if the simulation itself is accurate enough to reproduce the true physics of the system, it is difficult to say. However, it appears evident that quantitative estimates of the fluctuations induced by intra and inter-molecular motions in site energies and in couplings (and their cross-correlations) as those provided by detailed QM/MM approaches have to be included in the modeling of possible coherences and their effects on the efficiency of photosynthetic complexes. 


\section{ASSOCIATED CONTENT}

\section{Supporting Information}

A more detailed description of the methodology and additional data are provided in the supporting information. This material is available free of charge via the Internet at http://pubs.acs.org.

\section{AUTHOR INFORMATION Corresponding Author}

*E-mail: lucas.viani@for.unipi.it, carles.curutchet@ub.es, bene@dcci.unipi.it

\section{Notes}

The authors declare no competing financial interest.

\section{Acknowledgements}

The European Research Council (ERC) through the Starting Grant proposal n. 277755 (EnLight) is acknowledged by B.M. and L.V. . C.C. acknowledges support from the Ministerio de Economía y Competitividad of Spain through the Ramon' y Cajal program (ref. RYC-201108918) and computational resources provided by the Centre de Supercomputació de Catalunya. We thank Dr. Alexandra Olaya-Castro for the helpful discussion and the interesting suggestions.

\section{REFERENCES}

(1) Cheng, Y.-C.; Fleming, G. R. Ann. Rev. Phys. Chem. 2009, 60, 241-62.

(2) Novoderezhkin, V. I.; Van Grondelle, R. Phys. Chem. Chem. Phys. 2010, 12, 7352-65.

(3) E. Collini, C. Curutchet, T. Mirkovic, and G. D. S. In Energy Transfer Dynamics in Biomaterial Systems; I. Burghardt, V. May, D.A. Micha, and E. R. B., Ed.; Springer, Berlin, 2009; p. 3.

(4) Fleming, G. R.; Scholes, G. D. Nature 2004, 431, 256-7.

(5) Scholes, G. D.; Fleming, G. R.; Olaya-Castro, A.; Van Grondelle, R. Nature chemistry 2011, 3, 763-74. 
(6) Engel, G. S.; Calhoun, T. R.; Read, E. L.; Ahn, T.-K.; Mancal, T.; Cheng, Y.-C.; Blankenship, R. E.; Fleming, G. R. Nature 2007, 446, 782-6.

(7) Lee, H.; Cheng, Y.-C.; Fleming, G. R. Science 2007, 316, 1462-5.

(8) Collini, E.; Wong, C. Y.; Wilk, K. E.; Curmi, P. M. G.; Brumer, P.; Scholes, G. D. Nature 2010, 463, 644-7.

(9) Ishizaki, A.; Calhoun, T. R.; Schlau-Cohen, G. S.; Fleming, G. R. Phys. Chem. Chem. Phys. 2010, 12, 7319-37.

(10) Panitchayangkoon, G.; Hayes, D.; Fransted, K. A.; Caram, J. R.; Harel, E.; Wen, J.; Blankenship, R. E.; Engel, G. S. Proc. Natl. Acad. Sci. U. S. A. 2010, 107, 12766-70.

(11) Scholes, G. D. J. Phys. Chem. Lett. 2010, 1, 2-8.

(12) Yu, Z.; Berding, M.; Wang, H. Phys. Rev. E 2008, 78, 050902.

(13) Nazir, A. Phys. Rev. Lett. 2009, 103, 146404.

(14) Fassioli, F.; Nazir, A.; Olaya-Castro, A. J. Phys. Chem. Lett. 2010, 1, 2139-2143.

(15) Chen, X.; Silbey, R. J. J. Chem. Phys. 2010, 132, 204503.

(16) Nalbach, P.; Eckel, J.; Thorwart, M. New J. Phys. 2010, 12, 065043.

(17) Ishizaki, A.; Fleming, G. R. New J. Phys 2010, 12, 055004.

(18) Abramavicius, D.; Mukamel, S. J. Chem. Phys. 2011, 134, 174504.

(19) Strümpfer, J.; Schulten, K. J. Chem. Phys. 2011, 134, 095102.

(20) Hennebicq, E.; Beljonne, D.; Curutchet, C.; Scholes, G. D.; Silbey, R. J. J. Chem. Phys. 2009, 130, 214505.

(21) Vlaming, S. M.; Silbey, R. J. J. Chem. Phys. 2012, 136, 055102.

(22) Huo, P.; Coker, D. F. J. Chem. Phys. 2012, 136, 115102.

(23) Olbrich, C.; Kleinekathöfer, U. J. Phys. Chem. B 2010, 114, 12427-37.

(24) Olbrich, C.; Strümpfer, J.; Schulten, K.; Kleinekathöfer, U. J. Phys. Chem. B 2011, 115, 758-64.

(25) Adolphs, J.; Müh, F.; Madjet, M. E.-A.; am Busch, M. S.; Renger, T. J. Am. Chem. Soc. 2010, 132, 3331-43. 
(26) Müh, F.; Madjet, M. E.-A.; Adolphs, J.; Abdurahman, A.; Rabenstein, B.; Ishikita, H.; Knapp, E.-W.; Renger, T. Proc. Natl. Acad. Sci. U. S. A. 2007, 104, 16862-7.

(27) Müh, F.; Madjet, M. E.-A.; Renger, T. J. Phys. Chem.. B 2010, 114, 13517-35.

(28) Curutchet, C.; Novoderezhkin, V. I.; Kongsted, J.; Muñoz-Losa, A.; Van Grondelle, R.; Scholes, G. D.; Mennucci, B. J. Phys. Chem.. B 2012.

(29) Curutchet, C.; Muñoz-Losa, A.; Monti, S.; Kongsted, J.; Scholes, G. D.; Mennucci, B. J. Chem. Theory Comput. 2009, 5, 1838-1848.

(30) Wilk, K. E. Proc. Natl. Acad.Sci.U.S. A. 1999, 96, 8901-8906.

(31) Doust, A. B.; Marai, C. N. J.; Harrop, S. J.; Wilk, K. E.; Curmi, P. M. G.; Scholes, G. D. J. Mol. Biol. 2004, 344, 135-53.

(32) Doust, A. B.; Wilk, K. E.; Curmi, P. M. G.; Scholes, G. D. J. Photochem Photobio. A: Chem. 2006, 184, 1-17.

(33) Novoderezhkin, V. I.; Doust, A. B.; Curutchet, C.; Scholes, G. D.; Van Grondelle, R. Biophys. J. 2010, 99, 344-52.

(34) Curutchet, C.; Kongsted, J.; Muñoz-Losa, A.; Hossein-Nejad, H.; Scholes, G. D.; Mennucci, B. J. Am. Chem. Soc. 2011, 133, 3078-84.

(35) Zerner, M. C. In Reviews in Computational Chemistry; Lipkowitz, K. B.; Boyd, D. B., Eds.; Wiley VCH: New York, 1994; p. 313.

(36) Ichiye, T.; Karplus, M. Proteins 1991, 11, 205-17.

(37) Hünenberger, P. H.; Mark, a E.; Van Gunsteren, W. F. J. Mol. Biol. 1995, 252, 492-503.

(38) Lange, O. F.; Grubmüller, H. Proteins 2006, 62, 1053-61.

(39) Kraskov, A.; Stögbauer, H.; Grassberger, P. Phys. Rev. E 2004, 69, 1-16.

(40) Khan, S.; Bandyopadhyay, S.; Ganguly, A. R.; Saigal, S.; Erickson, D. J.; Protopopescu, V.; Ostrouchov, G. Phys. Rev. E 2007, 76, 026209.

(41) Olbrich, C.; Strümpfer, J.; Schulten, K.; Kleinekathöfer, U. J. Phys. Chem. Lett. 2011, 2011, 1771-1776.

(42) Kubar, T.; Kleinekathöfer, U.; Elstner, M. J. Phys. Chem. B 2009, 113, 13107-17.

(43) Kolli, A.; O’Reilly, E. J.; Scholes, G. D.; Olaya-Castro, A. J. Chem. Phys. 2012, 137, 174109 


\section{Figures}

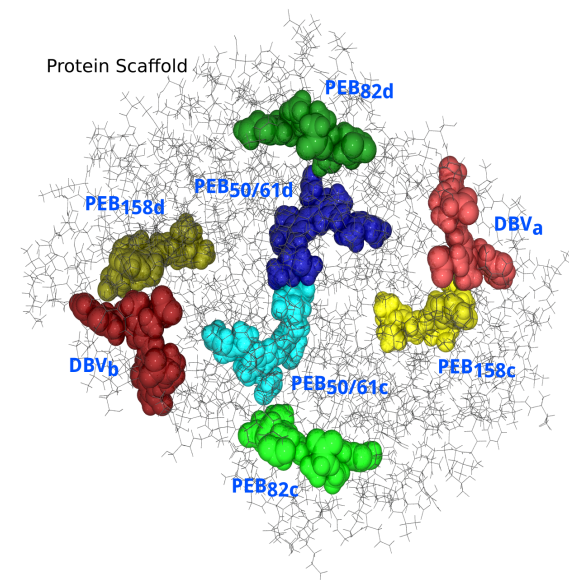

(a)

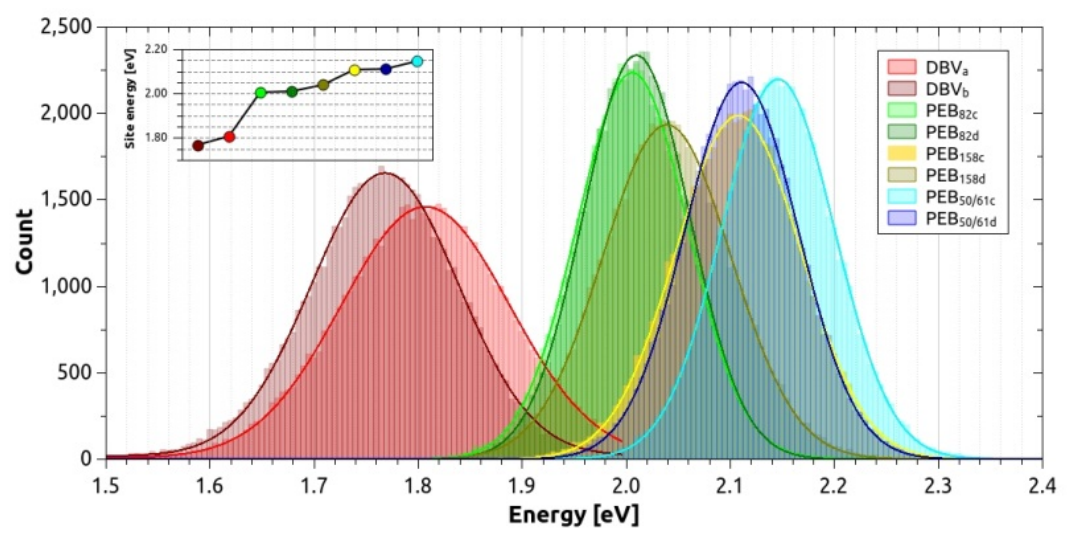

(b)

Figure 1: (a) Structure of the PE545 light-harvesting complex and a detailed view of the eight light-absorbing bilin molecules. (b) Distribution of the site energies of the chromophores computed using the MD-QM/MMPol method. The inset shows the site energy value at the center of the gaussian for each pigment. 


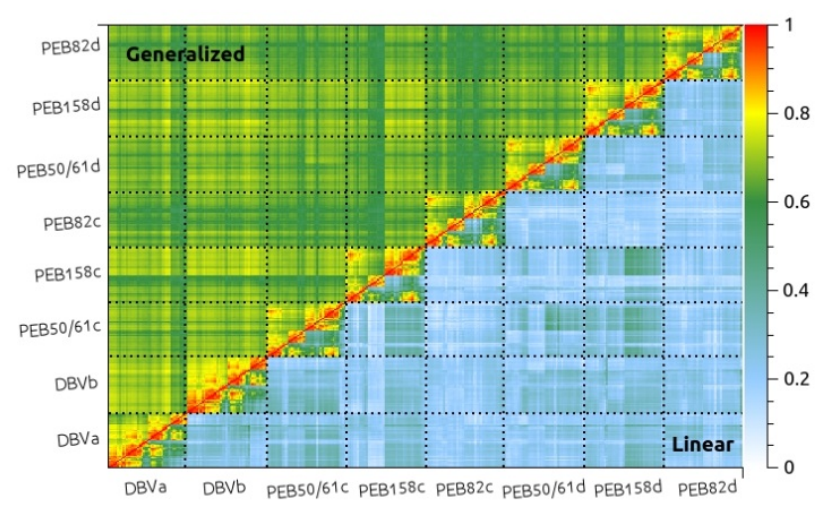

(a)

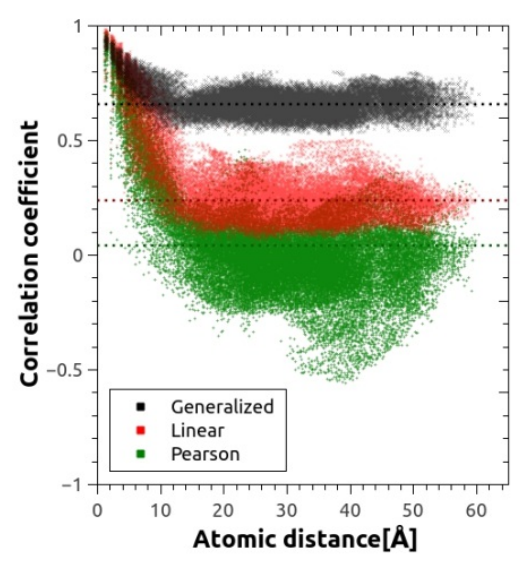

(b)

Figure 2: Correlations of spatial fluctuations between pairs of atoms for the GMI (upper triangle) and LMI (lower triangle) methods (a) and their distance dependence (b) together with the absolute Pearson coefficients. All the atoms of a single molecule are grouped together and no hydrogen atoms were included. In this representation, the rectangles placed along the diagonal show the intramolecular correlation pairs and the remaining ones the intermolecular correlation pairs. The dotted lines in (b) indicate the mean correlation coefficient among atoms with interatomic distance larger than $15 \AA$. 


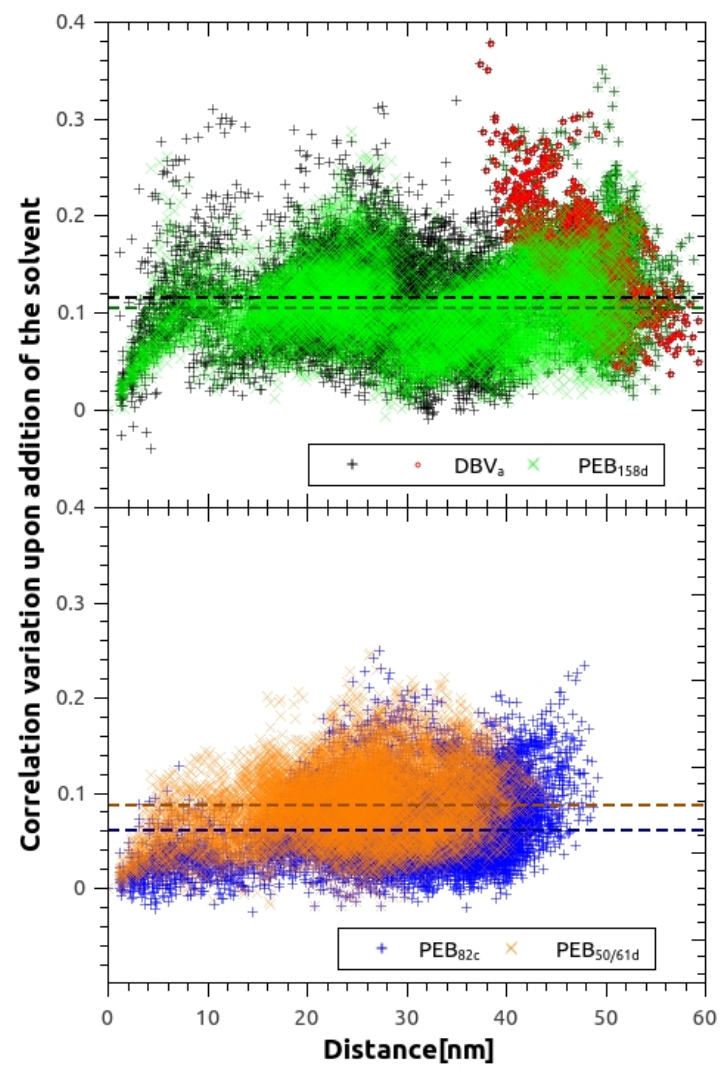

Figure 3: Variation of the correlation upon addition of the solvent for the chromophores $\mathrm{DBV}_{\mathrm{A}}$, $\mathrm{PEB}_{158 \mathrm{D}}, \mathrm{PEB}_{82 \mathrm{C}}$, and $\mathrm{PEB}_{50 / 61 \mathrm{D}}$. The dashed lines indicate the mean value of the distributions. The red symbols highlight the variations of the correlation between the $\mathrm{DBV}_{\mathrm{A}}$ and the $\mathrm{DBV}_{\mathrm{B}}$ molecules as a subset of the black symbols, which correspond to all correlations involving $\mathrm{DBV}_{\mathrm{A}}$. The mean intermolecular distances averaged over all frames are shown in Table $\mathrm{S} 1$. 


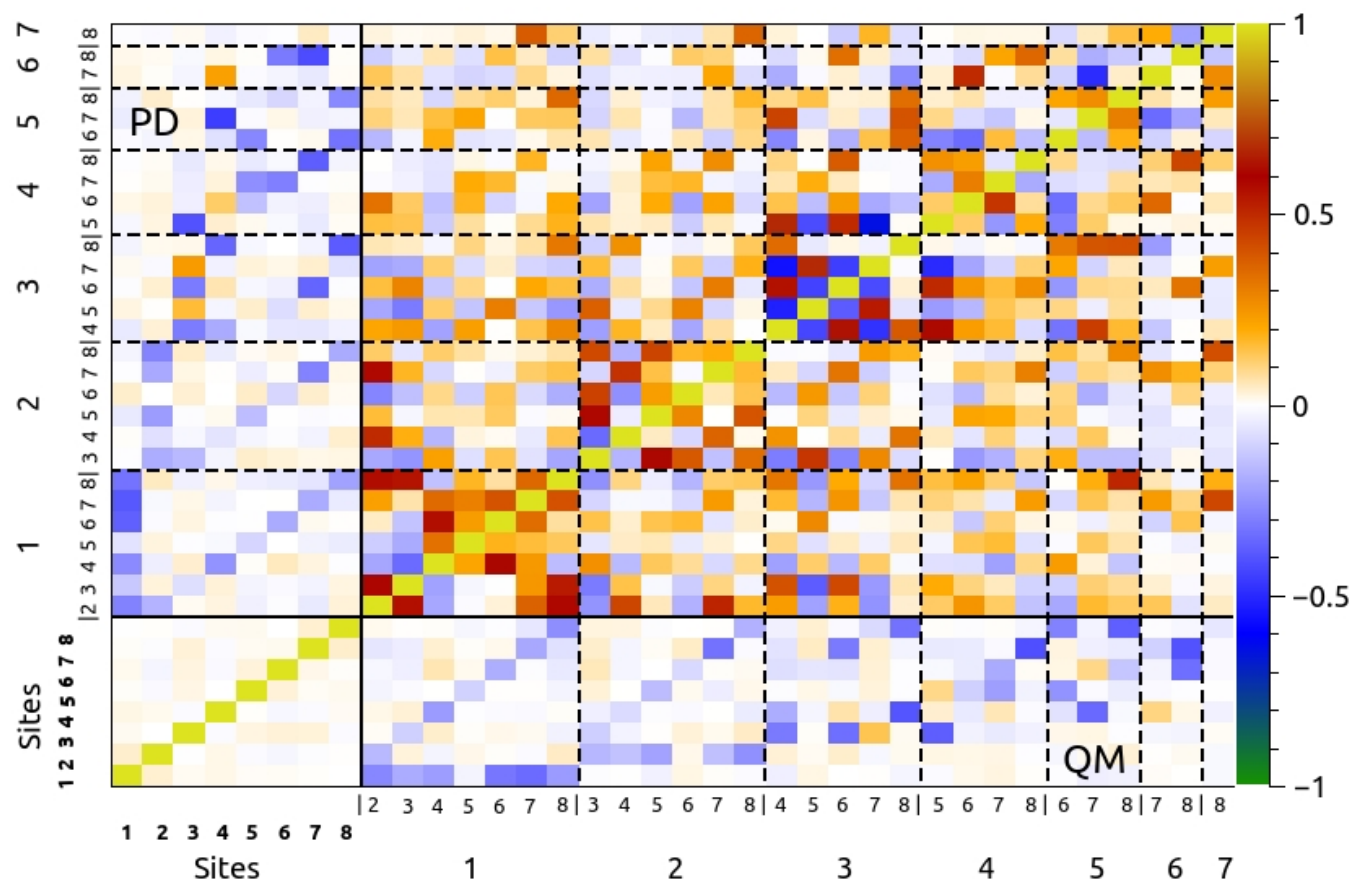

Figure 4: Correlation coefficients between the fluctuation of the site energies and excitonic couplings computed using the point dipole, PD, (upper triangle) and quantum chemistry, QM, (lower triangle) approaches. The chromophores are labeled from 1-8 in the following order: $\mathrm{DBV}_{\mathrm{A}}, \mathrm{DBV}_{\mathrm{B}}, \mathrm{PEB}_{50 / 61 \mathrm{C}}, \mathrm{PEB}_{158 \mathrm{C}}, \mathrm{PEB}_{82 \mathrm{C}}, \mathrm{PEB}_{50 / 61 \mathrm{D}}, \mathrm{PEB}_{158 \mathrm{D}}$, and $\mathrm{PEB}_{82 \mathrm{D}}$, respectively. 


\section{GRAPHICAL TABLE OF CONTENTS}

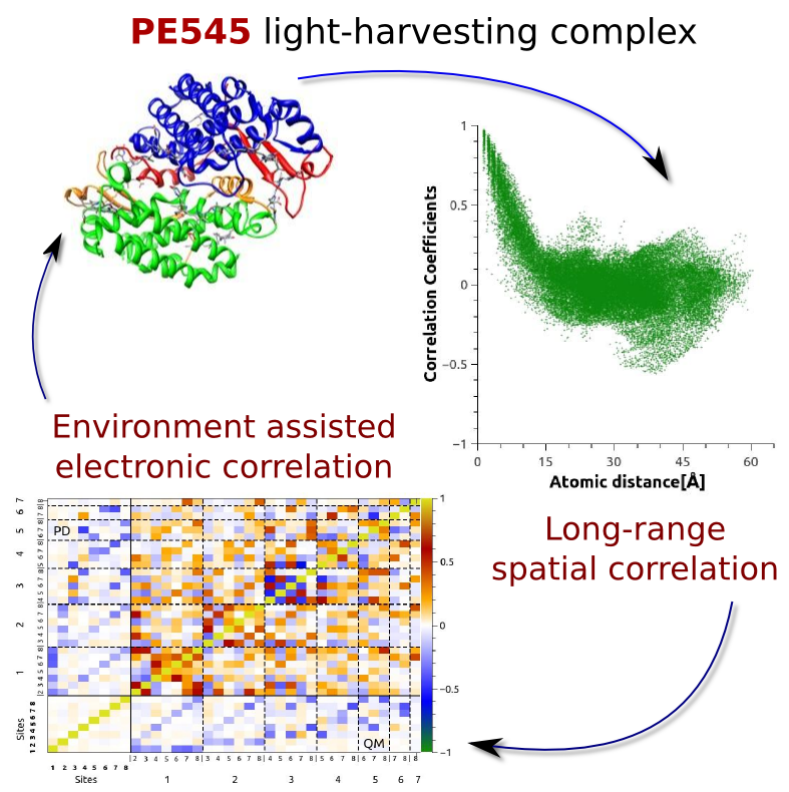

